\title{
UNA APORTACIÓN A LA TEMÁTICA HUMANÍSTICA EN INVESTIGACIÓN ENFERMERA: LA REVISTA CULTURA DE LOS CUIDADOS
}

\author{
José Siles GonZález \\ Departamento de Enfermería \\ Universidad de Alicante
}

\section{RESUMEN}

Este trabajo tiene el objetivo de describire el marco en el cual nace la revista "Cultura de los Cuidados". Asimismo, se valora la influencia de las revistas nacionales e internacionales de temática humanística.

Palabras clave: Cultura y enfermeria, historia de la enfermeria.

\section{A THEMATIC CONTRIBUTION TO HUMANISTIC IN NURSING RESEARCH: CULTURE OF CARES}

\section{ABSTRACT}

This work has the objective of describing the context in which the journal Cultura de los Cuidados is born. Also, the influence that international and national journals of humanistic topic has in this fact is valued

Keywords: Culture and nursing, nursing history.

\section{INTRODUCCIÓN}

La historia de la enfermería no surge cuando se inician los primeros cuidados en las sociedades primitivas, ni en la edad antigua, la medieval, o con el Renacimiento; ni siquiera nace al amparo del racionalismo ilustrado del siglo de las luces, o en el siglo XIX. La historia de la enfermería, es decir, los primeros estudios sobre el fenómeno de los cuidados (actividades, ideas, hechos), tiene su origen en la segunda mital del siglo XIX como con- secuencia de la transformación progresiva de los esquemas sociales, culturales e ideológicos responsables de dilucidar aquellos fenómenos susceptibles de convertirse en objeto-problema histórico. Los primeros estudios históricos sobre la enfermería están enfocados según la mentalidad de la época en la que surgen. La historiografía enfermera se inicia a partir del trabajo que un grupo de historiadores anglosajones realizaron durante la segunda década del siglo XIX. Estos estudios, con todas las virtudes y los defectos de la historiografía de la época, constituyen la raíz de la historia de la enfermería. Las razones de que esto haya sido así y que, sin embargo, en paises donde la enfermería ha se ha desarrollado antes, más y mejor, como es el caso de los países mediterráneos adalides del contrarreformismo (España, Portugal, Francia, Italia) hay que buscarlas, por un lado, en el poder configurador de la paradoja: tras el vacío asistencial provocado por el reformismo, las necesidades de los países reformistas en materia de cuidados de enfermería eran mucho más acuciantes e impostergables y esta situación de deterioro y dejadez influyó determinantemente en el nacimiento de la enfermería profesional, mientras que en los países católicos del sur de Europa los cuidados de enfermería siguieron vinculados durante mucho tiempo a la vocación religiosa. La abundancia de fuentes, tales como manuales de enfermería donde se reseñan los conocimientos de los enfermeros-as de la época, los registros y reglamentos hospitalarios que dan cuenta de las actividades de enfermeras, 
- Estudios históricos sobre instituciones sanitarias civiles, militares y/o religiosas (trabajos que suelen reflejar, además de los aspectos de la evolución administrativa del centro, la vida cotidiana de los profesionales del mismo.

- Power con su bosquejo histórico sobre los hospitales publicado en 1866 fue uno de los pioneros en la historia hospitalaria. Carlisle realizó en 1893 un estudio sobre el famoso hospital neoyorquino "Bellevue" estimulado por la sociedad de alumnos de dicho centro (CARLISLE, 1893). Cuatro años más tarde, Morton publicó su historia del hospital de pensilvania (MORTON, 1897) en la que recogía las características esenciales que marcaron la evolución de dicho centro desde su fundación. Pero es quizás el trabajo de Rathbone "Sketch of the history and progress of distric nursing from its commencement in the year 1859 to the present" el que trata de forma más específica el fenómeno enfermero centrandose para ello en la evolución histórica del distrito enfermero de Nueva York. Holland escribió en 1895 un libro en el que reflejó las proezas y el espíritu de sacrificio demostrado por las enfermeras militares.

- Longmore, al escribir en 1883 una historia comparada tomando como referencia las distintas formas de organizar y desarrollar los servicios sanitarios del ejército británico y el francés durante la guerra de Crimea, se convirtió en un importante antecedente de la metodología comparada en la historia, metodología que e ha sido aplicada a la educación, la sociología, la psicología, etc.

- Estudios históricos y antropológicos en los que se pone de manifiesto el carla división sexual del trabajo, asignándole en dicho reparto las tareas de cuidar a los enfermos, la higiene de los miembros de la tribu o la familia a las mujeres (MAS$S O N, 1894)$. En la primera tesis que se realiza en España sobre la relación entre la historia de la enfermería y la configuración de la disciplina enfermera queda patente que, mientras que el número de publicaciones de hisrtoria de enfermería ha ido aumentando, en términos relativos, la proporción de las mismas ha seguido una tendencia decreciente.

Ya a mediados del siglo XX el nivel de la historia de la enfermeria anglosajona evidenciaba notables avances en su consolidación y las preocupaciones metodológicas empezaban a ocupar un espacio importante en las revistas de enfermería inglesas y americanas las cuales se hacian eco de las reflexiones historiográficas de historiadores de enfermería cada vez más y mejor formados (AUSTIN, 1958). Posteriormente se publicaron monografías dedicadas a la cuestión metodológica en historia de la enfermeria (FITZPATRICK, 1978), centrándose incluso en las repercusiones que la investigación histórica podía tener en la investigación enfermera (KRUMAN, 1985), y en el mundo de las ideas en general (HAMILTON, 1993) y el desarrollo intelectual de los enfermeros-as en particular (HAMILTON, 1991). Los historiadores de la enfermeria de estos países, al comprender que el desarrollo de la enfermería se había producido como consecuencia de la incorporación de la mujer en el sistema educativo y laboral, han prestado una especial atención al paralelismo existente entre el proceso de liberación de la mujer y el desarrollo de la enfermería (LEWENSON, 1990, 1993). La razón de la primacía historiográfica anglosajona -inteligencia aplicada a proceso histórico-, hay que buscarla en dos factores externos a la propia enfermería:

Reflexiones teóricas resultantes de los esfuerzos de los pueblos por resolver sus problemas:

Feminismo (la mujer adquiere una mayor relevancia social, fundamentalmente porque toma conciencia histórica de su problemática).

$\square$ Las actividades domésticas -entre las que ocupa un lugar destacado los cuiados - van a experimentar un proceso de profesionalización en 
el marco de la sociedad reformista mejor preparada para el capitalismo.

Lucha de clases.

En mucha mayor medida que las mujeres, los obreros y la clases proletaria, en general, toman conciencia histórica e inician la lucha reivindicando una serie de cambios de formas de vida entre las que destacan las mejoras en su educación y en la asistencia social y sanitaria para acabar con el analfabetismo y la discriminación en situaciones de salud-enfermedad.

A la luz de todos los cambios que se van produciendo en la sociedad se revisan las antiguas fuentes aplicando los nuevos esquemas mentales y que perfeccionan la interpretación de aquellas (hermenéutica).

Nuevo concepto de salud, enfermedad y de enfermería.

- Evolución de las fuentes históricas como resultado de la actividad contradictoria y transformadora de la sociedad.

Evolución de técnicas de análisis de las herramientas, los gestos técnicos y las relaciones sociales pertinentes para la historia de la enfermería.

Análisis de ritos, ideologías y mitologias manifestadas en la vida cotidiana. Y que están muy vinculados a los cuidados de salud.

\section{CONTEXTO HISTÓRICO EN EL QUE SURGE CULTURA DE LOS CUIDADOS}

Ni cultura de los Cuidados ni cualquier otra revista de similares características hubiera sido posible sin la existencia previa de otras muchas publicaciones que le precedieron. En el ámbito internacional hay que destacar la labor de la enfer- mería estadounidense. La revista de la enfermería norteamericana más antigua fue creada en 1888 ("The Trained Nurse and Hospital Review"). Pero la primer a revista en la que se publicaron con asiduidad trabajos históricos fue "The american Journal of Nursing" que aparece con el siglo XX, justo un año después de la creación de Consejo Internacional de Enfermería, y que bajo el estímulo de la eminente historiadora de la enfermería, Mary M. Roberts ${ }^{1}$ contribuyó al avance de la historia enfermera (DONAHUE, 1988). Sin embargo las revistas de historia de la enfermería y antropología no aparecen hasta la década de los setentaochenta del siglo XX y, en general, lo hacen propiciadas por grupos de historiadores de la enfermería que persiguen fines comunes: el mantenimiento de un foro donde poder intercambiar experiencias relativas a sus trabajos históricos, propiciar mecanismos de ayuda para la investigación histórica (becas, premios, congresos), y, en general, contribuir al desarrollo de la historia de la enfermería. La Asociación Americana para la Historia de la Enfermería se fundó en 1978 con el nombre original de "Sociedad Internacional de Historia de la Enfermeria". Entre sus actividades destacan la publicación de la revista oficial de la asociación con periodicidad anual, la "Nursing History Review", el boletín trimestral "Bulletin of the American Association for The history of Nursing", además de convocar dos premios de investigación histórica y una beca para nuevos investigadores ${ }^{3}$. Otra publicación muy relacionada con la historia de la enfermería, aunque centrada en los estudios antropológicos es la "Revista de Enfermería Transcultural" que aparece a finales de los ochenta

\footnotetext{
' Mary M. Roberts escribió una obra clave en la historia de la enfermeria americana: American nursing: history and interpretation. The MacMillan Co. New York.

2 La revista "Nursing History review» es la heredera de otras dos publicaciones que le precedieron: «Journal History of Nursing» (19851987), y Society of Nursing History Gazette (1985-1988).

'Las actividades y recursos de la «American Association for the history of Nursing» pueden consultarse en Internet en la dirección: http://users.aol.com/NsgHistory/AAHN.htlm. La dirección postal para recabar cualquier tipo de información es: PO BOX 90803/Washington, DC 20090-0803/Dr. Rosemary McCarthy. Executive secretary.
} 
también como consecuencia de las necesidades de difusión y vertebración científica de una asociación denominada "Sociedad de Cuidados Transculturales".

History of nursing group bulletin.

London: History of Nursing Group at the Royal College of Nursing,

1985-1989. - Continued by: History of Nursing Society journal. -

History of Nursing Society journal.

London: Royal College of Nursing, 19901995

3 times yearly (each volume covers 2 years). - Continues: History of

Nursing Group at the Royal College of Nursing bulletin.

Hasta finales de los ochenta, los historiadores de la enfermería españoles no tenían más opción que publicar sus trabajos en revistas de enfermería general. Es a raíz de la fundación de la revista Index de Enfermería ${ }^{+}$cuando se abre una puerta para aquellos que trabajan la historia de la enfermería y desean publicar sus investigaciones en una revista especializada en custiones antropológicas e históricas. La primera base de datos nacional de enfermería "Cuiden" surge también al amparo de esta revista y entre sus actividades destaca la organización de cursos de metodología cualitativa y técnicas de documentación.

Hiades es la primera revista española exclusivamente dedicada a la historia de la enfermería. Dicha publicación aparece en Agosto de 1994 propiciada por el esfuerzo del Grupo Andaluz para la Investigación de la Historia de la Enfermería (GAIHE)s. El número uno de Cultura de los
Cuidados. Revista de Enfermería y Humanidades ve la luz en Agosto de 1997. Dicha revista se caracteriza por su empeño en el desarrollo de la enfermería humanística en cuatro apartados fundamentales: fenomenología, antropología, historia y teoría enfermera (TABLA I).

\section{CULTURA DE LOS CUIDADOS: FILOSOFIAA Y CARACTERÍSTICAS.}

Tal como se proclama en su primera editorial, publicada en junio de 1997, Cultura de los Cuidados surge para contribuir al desarrollo del conocimiento fenomenológico, histórico, antropológico y de teoría y método de los cuidados enfermeros, constituyendo el conocimiento previo de las culturas el paso preliminar indispensable para cualquier tipo de abordaje investigador en estas materias. Incluso se busca la propia identidad en publicaciones internacionales de contenido socio antropológico y humanístico que, en contra de la tendencia generalizada en las revistas de enfermería y médicas españolas (normativizadas según normas de Vancouver), siguen las normas de la American Psychology Association. En consecuencia, la revista se estructura en cuatro apartados:

-Fenomenología, en el que tienen cabida tanto artículos como narraciones en las que se relatan experiencias de personas - profesionales y no profesionales- que han pasado por diferentes situaciones dentro del variado intervalo "vida-salud". En esta línea de reforzamiento de la instrumentación literaria como fuente de conocimiento de la realidad humana en el contexto de los cuidados hay que interpretar la convocatoria anual de los premios "Vida y Salud" de narrativa.

\footnotetext{
+ El impulsor de la revista «Índex de Enfermería» es Manuel Amezcua, enfermero y antropólogo a quien se debe la primera publicación española con un sesgo temático idóneo para la antropología de los cuidados y la historia de la enfermería.

'Este grupo ha realizado una labor ejemplar en el proceso de desarrollo de la historia de la enfermería y está compuesto por Manuel J. García Martínez, Antonio C. García Martínez y Juan I. Valle Racero.
} 


\section{TABLA I}

\section{REVISTAS DE ENFERMERÍA ESPECIALIZADAS EN TEMAS HISTÓRICOS, ANTROPOLÓGICOS Y HUMANISTICOS}

\author{
ESPAÑOLAS \\ - HÍADES (1994) \\ (http//www.arrakis.es/-híades) \\ -INDEX DE ENFERMERIA \\ (1988) (http//www.doc6.es/index
}

- CULTURA DE LOS CUIDADOS (1997)

(http://enfe.ua.es/cultura/indice.html)

\author{
EXTRANJERAS
}

-JOURNAL OF TRANSCULTURAL NURSING (1989)

(http://www.culturenurse.org/main/mainindex.html)

-BULLETIN AMERICAN ASSOCIATION FOR

THE HISTORY OF NURSING

-NURSING HISTORY REVIEW. (1993)

(http://users.aol.com(NsgHistory/NHRTOC.html)

-JOURNAL OF NURSING HISTORY.

-SOCIETY FOR NURSING HISTORY GAZETTE (1985-1988)

Fuente: Bases de datos (Cuiden, Medline: IM, INI); Directorio revistas biomédicas españolas, Centro de Documentación e informática biomédica, Universidad de Valencia.

—La sección de Historia está dedicado a potenciar la identidad del colectivo enfermero como grupo socioprofesional como paso previo para la toma de conciencia indispensable para influir en la su evolución y desarrollo.

—En el apartado antropológico, se pretende abrir un espacio para publicar los trabajos de antropología de los cuidados y refrendar, de esa forma, el carácter holístico tan vinculado a la naturaleza de la enfermería, constituyendo el estudio del hombre y su cultura un requisito ineludible.

- Teoría y método es una sección que pretende servir de foro para intercalar todos aquellos trabajos susceptibles de incrmentar el potencial vertebrador de la teoría y los métodos aplicables en cuidados.

Además se incluye una miscelánea en la que tienen cabida tanto la crítica de novedades científico-literarias, como la propaganda de eventos de interés humanístico para la enfemrería.
En el período 1997-1999, la temática de los trabajos publicados en Cultura de los Cuidados ha seguido una evolución regular manteniendo una cierta equiparación con diferencias porcentuales que no superan el 6\% (TABLAII). Así, se observa como el mayor porcentaje de publicaciones le corresponde al apartado temático "historia" (27\%), seguido por "Antropología" y "Fenomenología", ambos con el 26\%, siendo los trabajos relativos a "Teoría y método" los de menor porcentaje (21\%).

\section{LAS PRIMERAS MONOGRAFIAS EN LA HIS- TORIOGRAFÍA ENFERMERA}

Un breve repaso a la historiografía española señala claramente cómo en España hasta hace muy pocos años no se han realizado estudios históricos de enfermería, permaneciendo en un letargo poco o nada productivo, un período de digestión de las 
aportaciones anglosajonas. Esto es así, porque, aparte de las introducciones históricas insertadas de forma más que genérica en los manuales de Practicantes y Ayudantes Técnicos Sanitarios publicados a raíz de la aplicación de la Ley Moyano (1857) y los textos clásicos de la historia de la medicina que sirvieron de guía a los pioneros de la historia enfermera (GRANJEL, 1962; AlBARRACÍN, 1973, LAÍN ENTRALGO, 1982), los primeros trabajos históricos sobre enfermería se localizan en las décadas 1980-1990: Es durante estos años cuando se publican los trabajos de los denominados pioneros de la historia de la enfermería española quienes realizan sus aportaciones mediante enfoques sociohistóricos estudiando lá naturaleza de los cuidados y su vinculación como actividad social a la distribución sexual del trabajo (DOMÍNGUEZ, 1981, 1983, 1986); centrado en la evolución normativa de enfermeras, matronas, practicantes y sangradores (VENTOSA, 1981); o acometiendo un arduo y amplio trabajo recopilatorio para reconstruir la historia de la enfermría española e hispanoamericana (ESEVERRI, 1984) (TABLA III).
Con la llegada de los noventa la situación historiográfica de la enfermería inicia un período de vertebración mediante la agrupación de los historiadores de la disciplina en el "Seminario Permanente de Historia de la Enfermería" presidido por la profesora de la Complutense Francisca Hernández. Como consecuencia de esta toma de conciencia de los enfermeros por su historia, se organizan las primeras jornadas y congresos nacionales que sirven para que los historiadores presenten sus trabajos y puedan intercambiar experiencias relacionadas con el desarrollo historiográfico de la enfermería ${ }^{6}$. En la década de los noventa se publican una serie de manuales de historia de la enfermería centrados en la historia general sin olvidar los aspectos metodológicos de la disciplina (HERNÁNDEZ CONESA, 1995); enfocados a la historia de la enfermería española (CALVO, 1993; CASTRO, 1993; HERNÁNDEZ MARTíN, Ed; 1996; SILES, 1996a, 1999). En esta década empiezan a publicarse los primeros estudios regionales y locales: la enfermería en Castilla la Mancha (SÁNCHEZ, et al, 1996), la enfermería sevillana a través del colegio (GALLARDO, JALDÓN y VILLA,

\section{TABLA III}

\section{TRABAJOS PIONEROS EN LA HISTORIOGRAFIA ENFERMERA DURANTE LA DÉCADA DE LOS OCHENTA}

CARMEN DOMÍNGUEZ ALCÓN

FCO. VENTOSA ESQUINALDO

CECILIO ESEVERRI CHAVERRI
"Sociología y enfermería" (1981)

"La infermeria a Catalunya (1981)

"Los cuidados y la profesión de enfermería en España" (1986)

"Historia de la enfermería española" (1981)

"Historia de la enfermería española e hispanoamericana" (1984)

Fuente: SILES, J. (1996a) Pasado, presente y futuro de la enfermeria española: una perspectiva histórica y epistemológica. Consejo Valenciano de Enfermeria/ Fundación José Llopis, Alicante.

\footnotetext{
El primer Congreso Nacional de Historia de la Enfermeria tuvo lugar en Valencia y se presentaron trabajos de historiadores procedentes de toda la geografía española (Hernández Martín, F. De. 1996).
} 
1993), la enfermería almeriense en el conjunto de la colectividad sanitaria en la provincia de Almería (AMEZCUA, GERMÁN, HEIERLE, 1994), se publica incluso una tesis doctoral sobre la enfermería andaluza a través de la prensa regional (LASARTE, 1995)7. Otros autores han concentrado sus esfuerzos en el desarrollo de sectores específicos de la enfermería: estudios históricos sobre las matronas y la enfermería pediátrica (Mart..., sobre las órdenes religiosas y el rescate de manuales históricos (GARCIA MARTÍNEZ et al, 1992; VALLE RACERO et al, 1993).

\section{A MODO DE CONCLUSIÓN}

Concluyendo, se puede afirmar que el nivel de desarrollo de la producción científica dentro del ámbito humanístico y social (historia, fenomenología, antropología y teoría y método) sólo resulta posible establecerlo mediante el repaso bibliográfico; es decir, mediante el estudio de todas los trabajos de todas y cada una de las materias que convergen en el plano humanístico de los cuidados de enfermería tanto por su enfoque como por su contenido. Cuando ha aumentado el número de monografías de una temática determinada, también ha experimentado un notable ascenso en la publicación de revistas orientadas en la misma línea. Estas estimaciones de carácter bibliométrico no deben interpretarse exclusivamente desde el punto de vista cuantitativo, aunque parece lógico estimar un mayor número de publicaciones de una misma temática ha de redundar en un incremento de la calidad de las mismas. En España, revistas como Index, Híades y Cultura de los Cuidados han contribuido a abrir el camino a la investigación humanística en enfermería erigiéndose en canales de vertebración y difusión de trabajos históricos, fenomenológicos, antropológicos y de teoría enfermera.

\section{BIBLIOGRAFIA}

ALBARRACÍN, A. (1973) La titulación médica en España durante el siglo XIX. Cuadernos de Historia de la Medicina Española. XII:45-47.

ALBERDI, R. M. ${ }^{a}$ et al (1983) Conceptos de enfermeria. UNED; Madrid.

AMEZCUA, M, Germán, C, Heierle, C. (1994) Sanidad y colectividad sanitaria en Almería. El colegio de practicantes (1885-1945). Colegio Oficial de Enfermería de Almería, Almería.

ARÓSTEGUI, J. (1995) La investigación histórica: teoría y método. Crítica, Barcelona.

BRAUDEL, F. (1968) La historia y las ciencias sociales. Alianza, Madrid.

BUENO, G. (1978) "Reliquias y relatos: construcción del concepto de historia fenoménica". El Basislisco 1:5

BUNGE, M. (1985) La investigación científica. Ariel, Barcelona. BURKE, P. (ed.) Formas de hacer historia. Alianza, Madrid.

CALderhead, J. (1986) Pensamientos de los profesores y toma de decisiones. Servicio de Publicaciones Universidad de Sevilla, Sevilla.

CAMPO ALANGE, Ma (1993) Concepción Arenal. Círculo de Lectores, Barcelona.

CARASA, P.(1985) El sistema hospitalario español en el siglo XIX. Caja de Ahorros de Salamanca/Universidad de Valladolid, Valladolid.

—(1985) El sistema hospitalario español en el XIX Caja de Ahorros de Salamanca, Universidad de Valladolid.

CARDOSO, C.F.S (1982) Introducción al trabajo de investigación histórica. Crítica, Barcelona.

CARLSON, J.H./ CRAFT, C.A./ MCGUIRE, A.D. (1985) Diagnóstico de Enfermería. Pirámide, Madrid.

CARR, E. H. (1979) ¿Qué es la historia? Seix Barral, Barcelona. CARR, W. \& KEMMIS, S. (1988) Teoría critica de la enseñanza. Martínez Roca, Barcelona.

CHENEY, E. (ed.) (1899) Louisa May Alcot: her life, letters and journals. Brown \& Co., Boston.

COOK, E. (1913) The life of Florencia Nightingale. 2 vols, Macmillan, London.

COOK, S.H. (1991) Mind the theory/practice gap in nursing. Journal of Advanced Nursing 16: 1462-1469.

DE MiGUEL, J.M. a (1976) La Reforma Sanitaria en España. Cambio 16, Madrid.

-(1983) Estructura del Sector Sanitario. Tecnos, Madrid.

DE MIGUEL, J. M. ${ }^{a}$ Y DOMínGUEZ, C. (1983) Sociología y Enfermería. Pirámide, Madrid.

DOMINGUEZ CARMONA, M. (1988) Concepto de Salud y Enfermedad. Medicina Preventiva y Salud Pública, Salvat, Barcelona.

" Esta importante tesis doctoral fue dirigida por el doctor Francisco Herrera. 
-(1981) Là infermeria a Catalunya. Rol, Barcelona.

— (1985) Sociología y Enfermería. Pirámide, Madrid.

- (1986) Los cuidados y la profesión de Enfermería en España. Pirámide, Madrid.

DONAHUE, P. (1988) Historia de la Enfermería. Doyma, Barcelona.

DONOHUE EBEN, J. et al (1989) Dorothea Orem: Teoría del déficit de autocuidado. Modelos y teorías de Enfermería. Rol, Barcelona

ECKESTEIN, L. (1896) Nurses under monasticism. Cambridge University Press, Cambridge.

ESEVERRI, C. (1984) Historia de la Enfermería española e hispanoamericana. Salvat, Barcelona.

- (1994) Los hermanos de San Juan de Dios. Diputación Provincial de Palencia, Palencia..

- (1995) Historia de la enfermería española e hispanoamericana. Universitas, Madrid

FICKSEIN, J.L. (1994) Nursing. The Nurse's Archives. In Conservation Administration News, 57: 14-15.

FITZPATRICK, M.L. (Ed) Historical studies in nursing. Teacher College Press, New York.

GALLARDO, C, JALDÓN, E., VILLA, V. (1993) La Enfermería sevillana. El colegio y su historia: 1900-1930. Publicaciones del colegio de Enfermeria de Sevilla, Sevilla

GALLASTEGUI, I. (1981) La odontología española en el siglo XIX. Tesis doctoral. Universidad Complutense, Madrid.

GARCIA LOZANO, A. (1993) Histôria de L'Escola D’Enfermería "Creu Roja" de Barcelona. Escuela de Enfermería de la Cruz Roja, Barcelona.

GARCÍA MARTÍNEZ, A.C; GARCÍA MARTÍNEZ, M.J; VALLE RACERO, J.I. (1996/97) La imagen de la matrona en la Baja Edad Media. Hiades, 3-4: 62-68.

GARCIA MARTINEZ, M.J; GARCIA MARTÍNEZ, J; y VALLE RACERO, J.I. (1995) El modelo de enfermería de San Juan de Dios. Index, IV/14: 31-35.

GARCÍA MARTÍNEZ, A. (1990) Ciencias sociales y epistemología. PPU, Barcelona

- (1992) Presentación y análisis de la obra "Instrucción de Enfermeros" de Andrés Fernández, 1625. Aproximación de la enfermería española de los siglos XVI-XVII. Consejo General de Enfermería, Madrid.

GARCÍA MARTíneZ, M.J. (dir.)(1994) Híades. Revista de Historia de Enfermería. 1/Agosto

GRANJEL, L.S. (1962) Historia de la medicina española. Sayma, Barcelona.

HAMILTON, D. (1991) Intellectual history. Paper presented at the meeting of Fourth Invitational Conference on Nursing History: Critical issues affecting research and researchers, Philadelphia.

- (1993) The idea of history and the history of teh ideas. Image Journal Nursing Scholarship, 25/1: 25-50.
HERNÁNDEZ CONESA, J. (1995) Historia de la enfermería. Interamericana McGraw-Hill, Madrid.

- (1997) La construcción de la enfermería como disciplina científica: una apreciación desde la historia y sus fundamentos históricos. Ed. Microfilmada. Servicio de Publicaciones de la Universidad de Murcia.

HERNÁNDEZ, F. (Dir) (1995) La enfermería en el siglo XX. De oficio a profesión. Los momentos del cambio. I Jornada Nacional de Historia de la Enfermería. E.U. de Enfermería, Fisioterapia y Podología de la Universidad Complutense, Madrid.

-(dir.)(1996) Actas I Congreso Nacional de Historia de la Enfermería. E.U. de Enfermería, Fisioterapia y Podología de la Universidad Complutense/E.U. de Enfermería del Hospital General Universitario de Valencia, Valencia.

HERRERA, F. (1989) Dos manuales para la formación de practicantes de la Armada. Revista de Historia Naval, VII/25: $69-87$

-(1992-1993) Estudios sobre las profesiones auxiliares sanitaria en Cádiz Siglos XIX-XX, IX-X: 213-268

—La titulación del enfermero psiquiátrico en la II República española. Minutos Meranini, 1990167:4-8

HOLLAND, M.A. (1895) Our Army nurses. B.Wilkins \& Co., Boston.

KAHLER, E. (1989) El significado de la historia. Círculo de lectores, Barcelona.

KAVANAGH, J. (1852) Women of Christianity. D. Appleton Co., New York.

LAÍN ENTRALGO, P. (1982) Historia de la Medicina Salvat, Barcelona.

LASARTE, J.E. (1995) La prensa de las profesiones auxiliares sanitarias en Andalucia. Estudio socioprofesional (19161939). De. Microfilmada, Servicio de publicaciones de la Universidad de Cádiz, Cádiz.

LONGMORE, T. (1883) The sanitary contrasts of the British and French armies during the Crimean War. Griffin \& Co, London.

MARTÍNEZ SAN PEDRO, R. (1975) La enfermera en la historia de la medicina. Alicante.

- (1978) La matrona en la historia de la medicina. Alicante

MOLINA, T.M. (1973) Historia de la Enfermeria. Interamericana, Buenos Aires.

NIGHTINGALE, F. (1990) Notas sobre Enfermería. ¿Qué es y qué no es? Salvat, Barcelona.

ORTEGA Y GASSET, J.(1973) Dilthey y la idea de la vida. Kant, Hegel y Dilthey. Revista de Occidente, Madrid.

RADCLIFFE-BROWN, A.R. (1986) Estructura y función en la sociedad primitiva. Agostini, Barcelona.

RATHBONE, W. (1890) Sketch of the history and progress of distric nursing from its commencement in the year 1859 to the present. The Macmillan Co, New York. 
SANTO TOMÁS, M. (1994) Historia de la Enfermería Enfermería Fundamental. Masson, Barcelona: 3-129.

SILES, J. (1990) Evolución histórica de una profesión multidisciplinar: la Enfermería y la Salud Pública en la España Contemporánea (1868-1936). Alacant Sanitari, 7:15-18.

- (1994a) Evolución histórica del corporativismo de Enfermería. La influencia de los colegios en el proceso de socialización profesional. Index III/10: 11-15.

SILES, J. et al (1994b) Implicación de la estructura familiar en la jerarquización del sistema de necesidades de la comunidad: un ejemplo de investigación participativa. Rol 196: 25-33.

SILES, J. (1995) Estructura familiar y función social de la mujer en Alicante (1868-1936). Colección "Textos Universitarios" Consellería d'Educació i Ciència Generallitat Valencianal Instituto Cultura Juan Gil-Albert, Alicante.

SILES, J. y GARCIA, E. (1995a) Las características de los paradigmas científicos y su adecuación a la investigación en enfermeria. Enfermería Científica 160/161: 10-15.

- (1995b) Análisis documental sobre las fuentes para la historia de los hospitales de Alicante. I Congreso Nacional de Historia de la Enfermería, Valencia.

- (1995c) Estudio documental sobre la influencia de la Cruz Roja en la enfermería española (desde sus orígenes hasta la II República) Enfermería Clínica vol 5/5: 7-13.

SILES, J. (1996a) Pasado, presente y futuro de la enfermería en España: Perspectiva histórica y epistemológica. CECOVA/ Fundación José Llopis, Alicante.

- (1996b) Origen histórico de la profesionalización de los cuidados mentales: practicantes, enfermeras y visitadoras psiquiátricas. Enfermería Cientifica, 174-175: 49-53.

- (1996c) El movimiento reformista durante el siglo XIX: su influencia en la enfermería española. En: Hernández, F. (Cood) Historia de la enfermería en España. Síntesis, Madrid: 227-240.

- (1996d) El proceso de institucionalización de la enfermería española (1900-1936. En: Hernández, F. (Cood) Hiștoria de la enfermería en España. Sintesis, Madrid: 241-255.

- (1996e) Colegiación y profesionalización de la enrfermería en España. En: Hernández, Fi (Cood) Historia de la enfermería en España. Sintesis, Madrid: 269-80 -(1996f) Evolución histórica de la "industria de las nodrizas" en Alicante (1868-1936). Actas II Congreso de Historia Social de España: el trabajo a través de la historia Asociación española de Historia Social/ Fundación Pablo Iglesias. Córdoba: 367-374.

- (1996g) Enfermería y conflictos bélicos. Index de Enfermeria. V/15: 1-2

- (1998a) El eslabón biológico en la historia de los cuidados de salud. El caso de las nodrizas: una visión antropológica de la enfermería. Index de Enfermería, 11/20-21: 16-23.

SILES, J. et al (1998b) La doble imagen de la enfermería en el cine durante la guerra civil española. Rol de Enfermería, 244: 24-31

SILES, J. (1999a) Historia de la enfermería comunitaria en España. Un enfoque social, político, cientifico e ideológico de la evolución de los cuidados comunitarios. Index (Aceptado para su publicación el 13.11.98)

-(1999b) Historia de la Enfermería. Teoría y Método. Aguaclara, Alicante.

- (1999c) Historia de la Enfermería. Teoria y Método. Consejo de Enfermería de la Comunidad Valenciana/ Fudación José LLopis, Alicante.

SOURNIA, J.C. (1994) Santé et mémoire. La Gazette Archives, 167: $343-346$

THOMPSON, P. (1988) La voz del pasado. Historia Oral. Alfons el Magnànim, Valencia.

TOPOLSKI, (1985) Metodología de la Historia. Cátedra, Madrid.

TOWLER, J \& Bramall, J. (1997) Las comadronas en la historia y en la sociedad. Masson, Barcelona.

VALLE RACERO, J.I, GARCIA MARTÍNEZ, M.J, GARCIA MARTÍNEZ, A.C. (1993) Notas sobre la llegada a Filipinas de los hermanos de San Juan de Dios a comienzos del siglo XVII. Qualat Chabir. Revista de Humanidades, 1.

VATTIMO, G.(1990) La sociedad transparente. Paidós, Barcelona.

VENTOSA, F: (1984) Historia de la Enfermería española. Ciencia 3, Madrid.

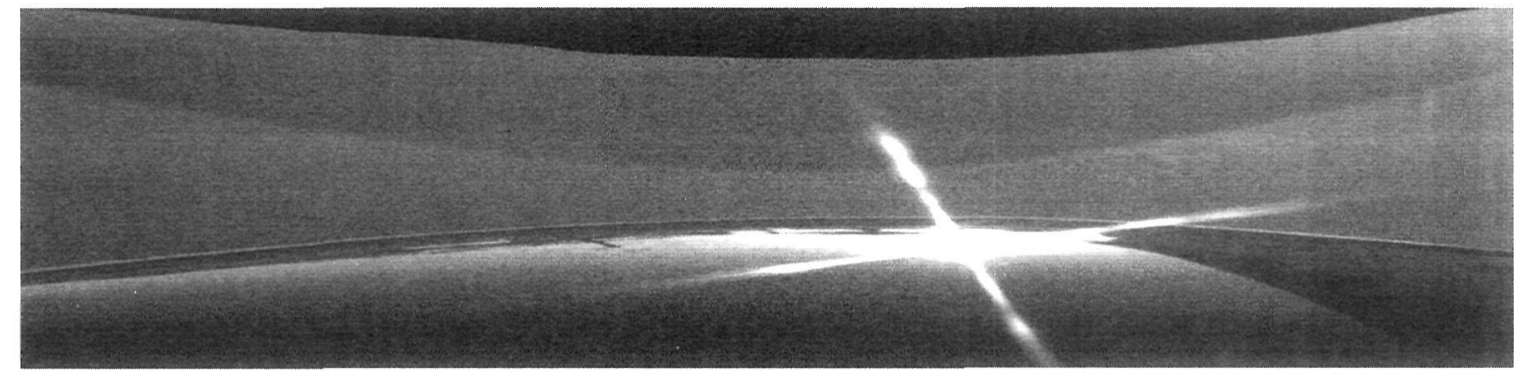

